\title{
Programa de Hepatopatias do Hospital da Fundação Santa Casa de Misericórdia do Pará - Infra-estrutura e epidemiologia clínica em 10 anos de atendimento
}

\author{
The Liver Diseases Program of the Hospital Santa Casa de Misericórdia do \\ Pará - Infrastructure and clinical epidemiology \\ in 10 years of attendance
}

\author{
Lizomar de Jesus Maués Pereira Moia ${ }^{1}$, Ivanete do Socorro Abraçado Amaral ${ }^{1}$, Simone Regina \\ Souza da Silva Conde ${ }^{1}$, Maria Sílvia Barbosa de Brito ${ }^{1}$, Esther Castello Branco Mello Miranda ${ }^{1}$, \\ Zilvana Pinheiro de Macedo ${ }^{1}$, Marialva Tereza Ferreira de Araújoㅇ, \\ Sâmia Demachki ${ }^{2}$ Manoel do Carmo Pereira Soares ${ }^{3}$
}

\begin{abstract}
RESUM0
O Programa de Hepatopatias do Hospital da Fundação Santa Casa de Misericórdia do Pará surgiu pela necessidade de prestar assistência a hepatopatas na região amazônica priorizando assistência qualificada, identificação das etiologias, seguimento clínico, e tratamento direcionado. Este trabalho visa descrever dados relativos à epidemiologia clínica, fatores etiológicos e análise histopatológica. Dos 1469 pacientes avaliados, através de exames clínicos, laboratoriais, endoscópicos e de imagem e/ou histopatológico, foram considerados hepatopatas crônicos 935 (63,6\%). Nesta casuística, a média de idade foi 50 anos, $666(71,2 \%)$ do sexo masculino e maior procedência de Belém. Os agentes etiológicos mais prevalentes foram alcoolismo (53,7\%) e hepatites virais (39,1\%). Biópsia hepática realizada em 403/935 (43,1\%), demonstrou hepatite crônica (34\%) e cirrose (34\%) na maioria das amostras. Conclui-se, portanto, que a doença hepática crônica na região é mais prevalente no sexo masculino, sendo o alcoolismo a principal etiologia e mais da metade dos casos se encontravam em fase avançada no momento do diagnóstico.
\end{abstract}

Palavras-chaves: Doença hepática crônica. Hepatite crônica. Hepatite viral.

\begin{abstract}
The Liver Diseases Program of the Hospital Santa Casa de Misericordia do Pará was create because of the need to attend patients with liver diseases of the Amazônia area, taking as priority to attend with quality, diagnosis of aetiologies, clinical following and specific treatment. This study aim to describe dates related to epidemiology, aetiologics agents and histopathologic analisys. One thousand sixthy nine patients were evaluated through medical, laboratory, endoscopic, ultrasound or computadorized tomography and histopathologic examination. Nine hundred thirty five (63,6\%) patients within 1469 patients were diagnose as chronic liver disease. The average age was 50 year, $666(71,2 \%)$ were male, and the most patients lived in Belem, State of Pará. The aetiologic agents most prevail were alcoholism (53,7\%) and viral hepatitis (39,1\%). Hepatic biopsy were done in $403(43,1 \%)$ within the 935 patients and the results showed chronic hepatitis (34\%) and chirrosis (34\%). In sumary the chronic liver disease in the amazon region is more prevail in male than female, the alcoholism is the principal aetiologie, and the most of these cases were diagnose in the severe phase.
\end{abstract}

Key-words: Chronic liver disease. Chronic hepatitis. Viral hepatitis.

\footnotetext{
1. Hospital Fundação Santa Casa de Misericórdia do Pará, Belém, PA, 2. Departamento de Patologia da Universidade Federal do Pará, Belém, PA, 3. Instituto Evandro Chagas da Secretaria de Vigilância em Sáude do Ministério da Saúde, Belém, PA.

Endereço para correspondência: Dra. Lizomar de Jesus Maués Pereira Móia. Seção de Hepatologia/Instituto Evandro Chagas. Av. Almirante Barroso 492 , 66090-000 Belém, PA.

Tel: 91 211-4461

e-mail: lizmoia@ amazon.com.br
} 
0 interesse pela estruturação das hepatopatias no Hospital da Fundação Santa Casa de Misericórdia do Pará (HFSCMPA) remonta os meados da década de 80, por meio de um Programa de Hepatopatias Crônicas, idealizado por clínicos do HFSCMPA e patologistas do departamento de Anatomia e Fisiologia Patológicas da Universidade Federal do Pará (UFPA). A implantação e implementação desse programa foi um desafio face às dificuldades da investigação diagnóstica, complexidade da etiopatogenia e abordagem terapêutica da doença hepática crônica ( DHC) . As dificuldades na investigação etiológica foram bastante minimizadas a partir de 1992 com a parceria do setor de hepatologia do Instituto Evandro Chagas (IEC) . Ao longo de 10 anos o Programa de Hepatopatias do HFSCMPA (PHHFSCMPA) necessitou transpor muitos obstáculos com 0 objetivo principal de estruturar um padrão diferenciado de atendimento clínico, laboratorial e terapêutico aos hepatopatas crônicos.

Os objetivos gerais do programa visam, dentre outros aspectos: a) oferecer um atendimento de referência especializado e de qualidade; b) informar sobre o número de pacientes que procuram atendimento por $\mathrm{DHC}$; c) identificar dentre esses os fatores condicionantes e determinantes da doença; d) fornecer dados histopatológicos; e) informar sobre ações de prevenção e tratamento das doenças hepáticas e, f) promover interação entre a assistência, ensino e pesquisa.

Atualmente, várias são as atividades conduzidas pelo programa, iniciando com as de assistência que objetivam diagnosticar e monitorar os estágios evolutivos da doença hepática, além de detectar e tratar precocemente as complicações clínicas e cirúrgicas, diminuindo dessa forma 0 número de internações e óbitos. Compondo a equipe multiprofissional e interdisciplinar, 0 acompanhamento ao hepatopata crônico conta com clínicos e cirurgiões geral e pediátrico, nutricionistas, enfermeiros, assistentes sociais e psicólogos.

Os pacientes matriculados no serviço recebem medicações básicas para o controle das complicações da DHC, assim como os medicamentos específicos para 0 tratamento das hepatites $B$, C, auto-imune e controle do pós-transplantado hepático. Os casos com indicação de transplante são encaminhados para centros de referência do País.

Enquanto respaldo para as atividades de pesquisa, o $\mathrm{PH}$ HFSCMPA serve de subsídios para estudos e pesquisas referentes a DHC na Amazônia, abrangendo linhas de investigação de epidemiologia clínica, sorológica e molecular, de correlação clínico-laboratorial-patológica e de resposta terapêutica. Em dez anos de existência, o programa originou uma vasta produção científica documentada em trabalhos publicados e apresentados em reuniões científicas ${ }^{13561011} 121819204142434446485253$, trabalhos de conclusão de curso $0^{29}{ }^{30} 50$ e dissertações de mestrado ${ }^{17} 40$.

Paralelamente, o PH-HFSCMPA participa da grade de ensino da graduação e pós-graduação das universidades Federal e Estadual do Pará, para os cursos de Medicina e Nutrição, Programas de Residência Médica e Mestrado em Medicina Tropical.

0 presente trabalho objetiva descrever resultados gerais de 10 anos de implantação do PH-HFSCMPA no que concerne os aspectos epidemiológicos e etiológicos da DHC na região.

\section{CASUÍSTICA E MÉTODOS}

No período de janeiro de 1992 a dezembro de 2002, cadastraram-se pacientes oriundos de vários serviços de saúde do Estado do Pará e outros vizinhos, como 0 Amapá e Maranhão, com média de 240 pacientes/mês, sendo atendidos mediante protocolo definido que englobou avaliação clínica, laboratorial, métodos endoscópicos e de imagem, e quando indicado a biópsia hepática. Esta última, realizada por agulha de Trucut dirigida por ultrassonografia, obedeceu às indicações e contra-indicações formais ao procedimento. 0 fragmento extraído foi encaminhado ao Departamento de Anatomia Patológica da UFPA, no qual se utilizou as colorações Cromotrope Azul de Anilina (CAB), reticulina de Gomori, Hematoxilina-Eosina (HE) e Perl's.

Os exames sorológicos para as hepatites A ( anti-HAV IgM e IgG) , B ( HBsAg, HBeAg, anti-HBc IgM, anti-HBc IgG, anti-HBe e anti-HBs), C (anti-HCV ) e delta (anti-HD) foram realizados no laboratório de hepatologia do IEC, usando técnicas imunoenzimáticas de kits comerciais. Neste mesmo laboratório, as amostras dos pacientes foram testadas para exames de biologia molecular, especificamente a reação em cadeia da polimerase (PCR) do HBV-DNA qualitativo e quantitativo e a PCR do HCVRNA qualitativo, quantitativo e genotipagem de ambos.

Complementando a investigação etiológica, quando necessários eram solicitados exames específicos como os testes de erros inatos do metabolismo, auto-anticorpos ( fator antinuclear - FAN, anticorpo antimúsculo liso - SMA, anticorpo antimicrossomal fígado-rim - LKM1, anticorpo antimitocôndria fração M2 - AMA), dosagens séricas de cobre, ferro, ferritina, saturação de transferrina e ceruloplasmina, dentre outros.

Os critérios considerados para definição de casos foram:

a) hepatite crônica não viral: pacientes sem positividade para $\mathrm{HBsAg}$, anti-HCV, anti-HD que apresentem dados clínicos, laboratoriais e/ou histopatológicos de hepatite;

b) hepatite crônica viral: pacientes com positividade para HBsAg, anti-HD ou PCR-RNA HCV, por um período superior a seis meses;

c) cirrose hepática: pacientes com ou sem positividade para HBsAg, anti-HCV ou anti-HD que apresentaram dados de insuficiência hepática e hipertensão porta ou resultado histopatológico compatível com cirrose;

d) carcinoma hepatocelular ( $\mathrm{CHC}$ ): pacientes que apresentem sinais e sintomas clínicos sugestivos, com diagnóstico por imagem compatível associado a níveis de alfafetoproteína acima de $400 \mathrm{ng} / \mathrm{ml}$ e/ou diagnóstico histopatológico;

e) etilismo: pacientes com ingestão de etanol diária mínima de $80 \mathrm{~g}$ para 0 sexo masculino e $40 \mathrm{~g}$ para 0 feminino, por um período superior a cinco anos.

Todos os atendimentos eram reportados para uma ficha de protocolo específica e inseridas no banco de dados do programa Epi Info (versão 2002). Os resultados foram analisados utilizando métodos estatísticos contidos no programa Biostat ( versão 2.0) . 
Os projetos de pesquisa oriundos do PH-HFSCMPA foram aprovados pelo Comitê de Ética em Pesquisa do IEC, conforme a resolução 196/1996 do MS/Brasil que prevê pesquisas envolvendo seres humanos.

\section{RESULTAD0S}

Em dez anos do PH-HFSCMPA (janeiro 1992 a junho 2002) , foram atendidos 1.469 pacientes, sendo 63,6\% (935) considerados hepatopatas crônicos. Nos casos com DHC, a média de idade foi 50 anos, com 71,2\% (666) do sexo masculino e 28,8\% (269) do feminino. Quanto à procedência, 67\% (616) eram procedentes de Belém, 30\% (276) de outros municípios do Pará e 3\% (24) de outros estados (Figura 1).

Dentre os 935 hepatopatas crônicos, foram identificados os agentes etiológicos em $799(85,5 \%)$, restando 136 $(14,5 \%)$ casos sem causa definida. Os agentes etiológicos prevalentes foram alcoolismo, hepatite $C$, hepatite $B$ isolados e/ou associados entre si (Figuras 2 e 3 ). Entre as etiologias menos freqüentes destacamos a hepatite delta com 1,5\% (6) e a hepatite autoimune $(2,8 \%)$.

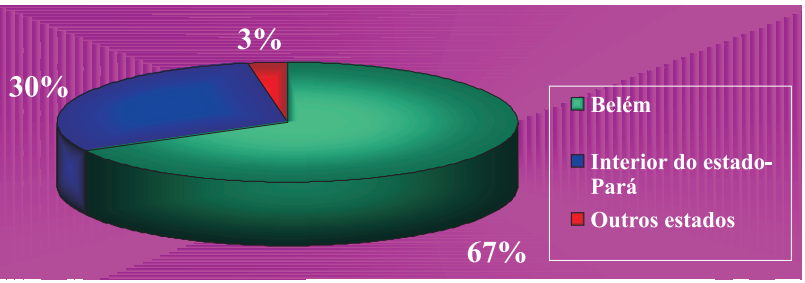

Doença Hepática ( $\mathrm{n}=935$ )

Distribuição quanto a procedência

Figura 1 - Programa de Hepatopatias do HFSCMPA. Distribuição quanto à procedência de 935 pacientes com doença hepática crônica.

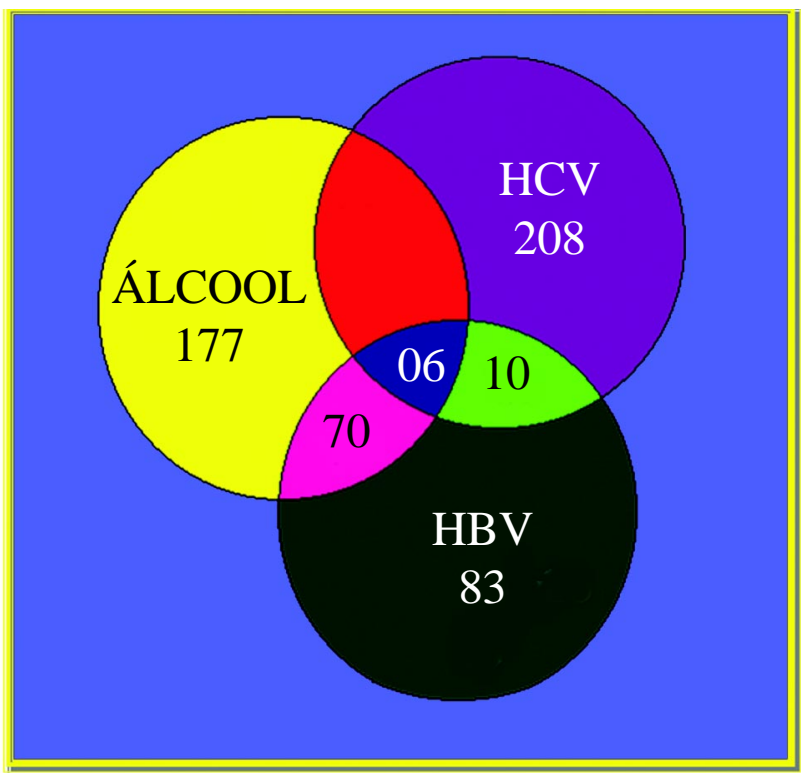

Figura 2 - Programa de Hepatopatias do HFSCMPA. Distribuição quanto as causas mais freqüentes de 935 pacientes com DHC.
A biópsia foi possível em 403/935 (43,1\%), onde os resultado histopatológicos demonstraram hepatite crônica em 137 (34\%), cirrose em 136 (33,7\%), hepatocarcinoma em 16 (4\%), fibrose hepática 15 (4\%), esteatose 28 (76\%), normal $19(5 \%)$, inconclusivo $12(3 \%)$, outros $40(10 \%)$, como demonstrado na Figura 4.

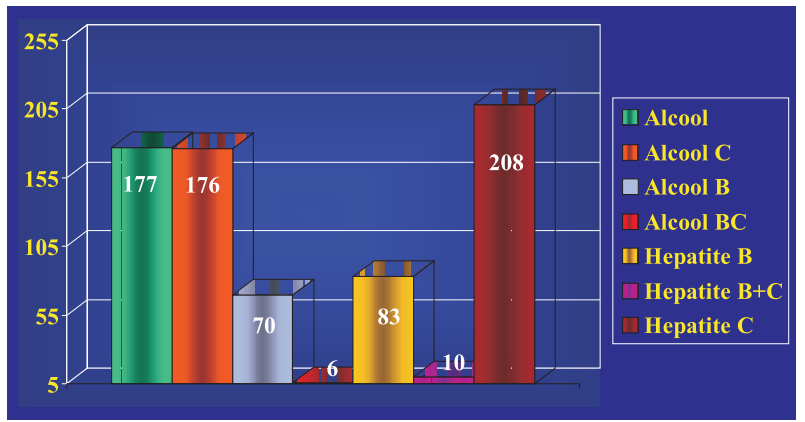

Figura 3 - Programa de Hepatopatias do HFSCMPA. Distribuição quanto as causas mais freqüentes de 935 pacientes com DHC.

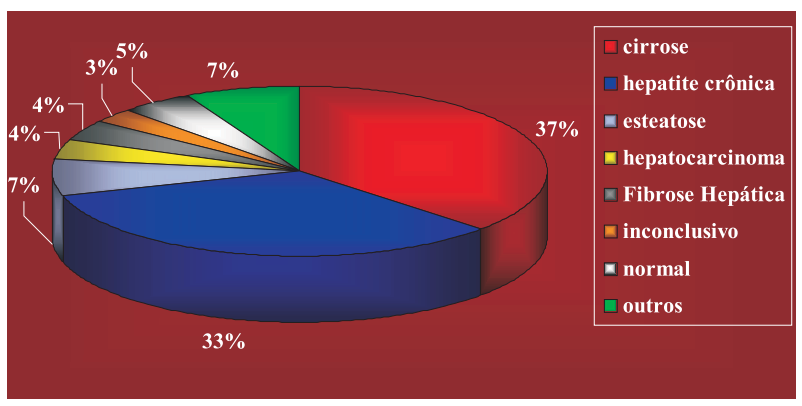

Outros: colestase; alterações discretas; inespecíficas; doença de Gaucher. Figura 4 - Diagnóstico Histopatológico de 403 pacientes com doença hepática crônica.

A correlação da etiologia e histopatologia hepática está contida Tabela 1, onde se pode observar que a presença de cirrose estava associada ao alcoolismo em 23/42 (54,7\%), à hepatite crônica C em 27/81 (27,7\%), à hepatite crônica B em 12/37 (32,4\%), ao anti-HD em 2/6 (33,3\%) e à hepatite autoimune em 8/17 (47\%).

Tabela 1 - Resultado do histopatológico de 403 pacientes com DHC relacionados aos agentes etiológicos.

\begin{tabular}{lrrrcr}
\hline & $\begin{array}{c}\text { Biópsias } \\
(\mathrm{n})\end{array}$ & Cirrose & $\begin{array}{c}\text { Hepatite } \\
\text { crônica }\end{array}$ & Hepatocarcinoma & $\begin{array}{r}\text { Outros } \\
\text { achados }\end{array}$ \\
Álcool & 42 & 23 & 5 & 2 & 12 \\
Álcool + HCV & 81 & 27 & 35 & 0 & 19 \\
Álcool + HBV & 29 & 9 & 8 & 4 & 8 \\
Álcool + HBV+ HCV & 2 & 1 & 1 & 0 & 0 \\
HBV & 32 & 12 & 11 & 8 & 6 \\
HCV & 109 & 27 & 51 & 0 & 31 \\
HCV/HBV & 2 & 1 & 2 & 0 & 0 \\
Hepatite autoimune & 17 & 8 & 9 & 0 & 0 \\
HDV & 6 & 2 & 3 & 1 & 0 \\
Outras etiologias* & 78 & 26 & 12 & 1 & 39 \\
Total & 403 & 136 & 137 & 16 & 114 \\
\hline
\end{tabular}

* Deficiência de $\alpha$ - 1 antitripsina, esteato-hepatite não alcoólica, doença de Gaucher. 


\section{DISCUSSÃO}

A maioria dos pacientes atendidos pelo PH-HFSCMPA eram hepatopatas crônicos, com etiologia identificada em 85,5\%, diferindo de estudos anteriores do mesmo programa, em que a causa indeterminada alcançava $23 \%$ dos $\operatorname{cas}^{18}{ }^{18}$. A redução do percentual de etiologia desconhecida no estudo atual pode ser explicada por várias razões: melhor suporte laboratorial, redução da quantidade da ingestão de álcool/dia considerada hepatotóxica, maior número de biópsias hepáticas realizadas definindo o diagnóstico em alguns casos como por exemplo esteatohepatite não alcoólica, deficiência de alfa-l-antitripsina, e ainda maior adesão dos pacientes às consultas facilitando a contínua investigação etiológica, resumindo no aumento e melhoramento nos métodos diagnósticos e investigativos da DHC ao longo da instalação do PH-HFSCMPA.

Os dados demográficos mostraram predomínio do sexo masculino com $71,2 \%$, a média de idade de 50 anos, e a procedência prevalente, Belém. Correlação entre esses dados serão objeto de estudos posteriores.

Entre as causas definidas, 0 alcoolismo apareceu em $53,8 \%$ dos casos, sendo como único fator $(22,2 \%)$ ou em associação com hepatite crônica C (22\%), hepatite crônica B $(8,9 \%)$ ou com ambas $(0,7 \%)$. É sabido que 0 etilismo se constitui importante causa de DHC no mundo e que sua associação com os vírus da hepatite $\mathrm{B}$ e $\mathrm{C}$ pioram 0 prognóstico, acelerando a progressão da doença ${ }^{24}$.

$\mathrm{Na}$ atual casuística, a hepatite crônica Cganha destaque com uma prevalência global de 48,2\%, isolado ou associado ao álcool. Desde sua descoberta em 1989 por Choo et al ${ }^{15}$, tem-se demonstrado que o HCV é um importante patógeno humano, com $3 \%$ da população mundial infectada por este vírus ${ }^{27}$. № Brasil, calcula-se a existência de 1,5 a 3,2 milhões de infectados ${ }^{29}$ e a Região Norte, em particular, possui prevalência de $2 \%$. Trabalhos apontam a hepatite crônica $\mathrm{C}$ como responsável por $70 \%$ das hepatites crônicas, $40 \%$ das cirroses e 30\% dos transplantes hepáticos, em países industrializados ${ }^{26}$.

0 HBV surge como terceira causa de DHC na população estudada, com 19,3\% de positividade para o HBsAg. Apesar da Amazônia ser considerada área de alta prevalência da infecção crônica pelo $\mathrm{HBV}^{37}$, reconhece-se que há heterogeneidade de achados nas diferentes populações amazônicas pesquisadas, predominando em áreas da Amazônia ocidental e em regiões rurais ${ }^{54} \mathrm{Com} 0$ advento da vacinação, observa-se um declínio da incidência e prevalência mundial da hepatite $B$ no mundo ${ }^{23}$. Porém ainda é considerada como problema de saúde pública, tendo 350 milhões de portadores crônicos e é responsável por um milhão de óbitos/ano ${ }^{22}$.

A co-infecção ou superinfecção pelo vírus da hepatite delta é uma ocorrência muito temida nas áreas onde a hepatite B é comum. Como demonstrado por Moia et al ${ }^{45}$ (2003), a maioria dos casos de hepatite delta se encontravam em fase avançada da doença, limitando a intervenção terapêutica.
Em muitos estudos ${ }^{1334}, 0$ alcoolismo e as hepatites crônicas virais $\mathrm{B}$ e $\mathrm{C}$ foram responsáveis pela grande maioria dos casos de DHC, à semelhança do que se observa no presente trabalho.

Coincidindo com casuísticas de outros estudos nacionais ${ }^{14}{ }^{28}{ }^{49}$ a prevalência da hepatite autoimune em uma casuística de DHCfoi em torno de 2,8\%, porém é uma importante etiologia devendo sempre ser lembrada nos casos em que vírus e álcool forem descartados e diante de uma população pediátrica e de adultos jovens, com predomínio de mulheres ${ }^{2132} 35$.

Os resultados histopatológicos de 403 pacientes biopsiados demonstraram 0 maior percentual de cirrose associado ao álcool sem infecção viral, à semelhança do que foi relatado ${ }^{44}$, porém conflitante com outras pesquisas onde a associação destes dois fatores aumenta a injúria hepática ${ }^{4248}$. Uma possível explicação para 0 fato seria a de que muitos pacientes deixaram de ser biopsiados por contra-indicações próprias da doença avançada, subestimando a prevalência de cirrose.

Nesta casuística, o hepatocarcinoma esteve associado com o HBV em maior proporção quando comparado com 0 álcool e a infecção pelo HCV. Sabe-se que há diferenças regionais quanto aos fatores etiopatogênicos do $\mathrm{CHC}$, sendo por exemplo fortemente ligado à infecção crônica pelo HBV na Amazônia, Formosa, Coréia e África do Sul 7333364047 , e ao HCV, no Japão , China, Espanha e Estados Unidos ${ }^{16} 255155$.

A DHC possui alta taxa de prevalência e morbiletalidade, tornado-se um sério problema de saúde pública mundial, nacional e em nossa região. O PH-HFSCMPA viabilizou um espaço de referência na rede pública, onde os profissionais e as instituições da área de saúde podem encaminhar os casos suspeitos para investigação, confirmação diagnóstica, acompanhamento clínico e terapêutico; assim como, permite a geração do conhecimento por meio do ensino e das pesquisas científicos, expandindo a visão sobre a DHC na região Amazônica, dentro das sua semelhanças, diferenças e particularidades.

\section{REFERÊNCIAS BIBLIOGRÁFICAS}

1. Acácio GJS, Moia LJMP, Conde SRSS, Barbosa MSB, Amaral ISA, Miranda ECBM, Soares MCP, Araújo MT, Demachki, Alves MM, Silva CAM, Cartágenes PRB. Etiologia da doença hepática crônica de uma casuística pediátrica de um hospital de referência. In resumos do XXXVIII Congresso da Sociedade Brasileira de Medicina Tropical, Foz do Iguaçu, p. 281, 2002.

2. Amaral EAA. Estudo da correlação da carga viral do RNA-HCV, dosagem de albumina, globulina, ALT e histopatologia em 40 pacientes anti-HCV positivos. Trabalho de Conclusão de Curso de Medicina, Universidade Federal do Pará Belém-PA, 1999.

3. Amaral ISA, Barbosa MSBB, Miranda ECBM, Moia LJMP, Conde SRSS, Araújo MTF, Soares MCP. Avaliação etiológica de 172 casos de doenças hepática crônica em um hospital geral, Belém-PA. In resumo XIII Congresso Brasileiro de Hepatologia, Florianópolis, p. 174, 1995.

4. Amaral ISA, Moia LJMP, Barbosa MSBB, Conde SRSS, Miranda ECBM, Moraes, MIF, Soares MCP, Araújo MT, Pinheiro, MC. Análise de 141 casos de doença hepática crônica alcóolica isolada ou associada à infecção viral. Gastroenterologia Endoscopia Digestiva 16:P0 112, 1997.

5. Amaral ISA, Rodrigues ALS, Soares MC, Araújo MTF, Demachki S, Silveira FA, Miranda ECB, Conde SRSS, Barbosa MSB, Moia LJMP. Hepatite tóxica por Croton cajucara-Estudo de quatro casos clínicos. In resumo do XV Congresso Brasileiro de Hepatologia, p. S70, 1999. 
6, Amaral ISA, Soares MCP, Boulhosa CF, Amaral EAA, Hidatidose policística por E. vogeli, Ilha do Marajó, Pará, Brasil. Revista Paraense de Medicina 14,2000 .

7. Amirudin R, Akil H, Akahane Y, Suzuki H. Hepatitis B and C virus infection in Ujung Pandang, Indonesia. Gastroenterology Japanese, 26: S184-S188, 1991.

8. Anderson S, Nevis CL, Green LK, EL-Zimaity H, Anand BS. Assessement of liver histology in chronic alcoholics with and without hepatitis $\mathrm{C}$ virus infection. Digestive. Disease. and Science, 46: 1393-1398, 2001.

9. Araújo IF, Rodrigues MJK, Albuquerque SSS, Cirrose Hepática: Perfil clínicoepidemiológico de 326 casos do PHC- HFSCMPA no período de janeiro de 1992 a junho de 2001. Trabalho de Conclusão de Curso de Medicina, Universidade Federal do Pará, Belém-PA, 1999

10. Araújo MTF, Batista HCN, Silva VC, Paixão NA, Pinheiro MCN, Conde SRSS, Barbosa MSB, Amaral ISA, Miranda ECBM, Soares MCP, Crescente AB. Estudo histopatológico de biópsias hepáticas em pacientes portadores de vírus C. In: resumos do I congresso Norte de Infectologia, Belém, p. 37, 1999

11. Barbosa MSB, Conde SRSS, Amaral ISA, Miranda ECBM, Moia LJMP, Soares MCP, Araújo MTF. Estudo clínico e laboratorial em 35 casos de doença hepática crônica anti-HCV poitivos. In resumos do XIII Congresso Brasileiro de Hepatologia, Florianópolis, p. 153, 1995.

12. Barbosa MSB, Conde SRSS, Moia LJMP, Amaral ISA, Miranda ECBM, Moraes MIF, Soares MCP, Araújo MTF, Pinheiro MCN. Estudo comparativo da transmissão pós-transfusional e esporádica em hepatopatas crônicos antiHCV positivos. In: resumos do XIV Congresso Brasileiro de Hepatologia, Goiás, p. 2000, 1997.

13. Bellentani S, Tiribelli C, Saccoccio G, Sodde M, Frade N, Martin C, Crstianini $G$ and Dionysis study Group. Prevalence of chronic liver disease in the general population of northern Italy: The Dionysos Study. Hepatology 20: 1442-1449, 1994

14. Cançado, ELR. Hepatite auto-imune. In: Silva LC, Hepatites agudas e crônicas, 2a edition. Sarvier, São Paulo p. 218-243,1995.

15. Choo QL, Kuo GAJ, Overby LR, Bradley DW, Houghton M. Isolation of a DNA clone derived from a blood borne nonA nonB viral hepatitis genome. Science244: 359-362, 1989.

16. Chuang WL, Chang WY, Lu, SN. The role of hepatitis B and C viruses in Hepatocellular carcinoma in a hepatitis B endemic area. A case-control study Cancer, 69: 2052-2054, 1992.

17. Conde SRSS. Prevalência dos genótipos e mutante pré- core A- 1896 do vírus da hepatite B e suas implicações clínicas, em uma população da Amazônia Oriental. Dissertação apresentada ao curso de Mestrado em Medicina Tropical da Universidade Federal do Pará, Belém-Pará, 2002.

18. Conde SRSS, Amaral ISA, Barbosa MSB, Miranda ECBM, Amaral EAA, Soares MCP, Araújo MTF, Demachki, S. Estudo etiológico de 459 casos de Doenças hepática crônica em Belém- Pará. In resumos do I Congresso Paraense de Gastroenterologia, Belém, p. 30, 1999.

19. Conde SRSS, Moia LJMP, Barbosa MSB, Amaral ISA, Miranda ECBM, Soares MCP, Araújo MT, Demachki S, Gomes M, Pinho JRR, Bertolini DA. Prevalência dos genótipos do vírus da hepatite B e sua correlação com apresentação clínica - epidemiológica, em uma população da Amazônia Oriental. In: resumos do XVI Congresso Brasileiro de hepatologia, Vitória, p. s30, 2001.

20. Conde SRSS, Moia LJMP, Barbosa MSB, Araújo MTF, Soares MCP, Amaral ISA. Hepatite autoimune entre hepatopatas crônicos de um hospital geral, Belém-PA. Revista Paraense de Medicina 12, 1998.

21. Czaja A and Carpenter HA. Validation of scoring system for diagnosis of autoimmune hepatitis. Digestive Diseases and Sciences 41: 305-314, 1996.

22. Davey S. State of the world's vaccines and immunization. Geneva: World Health Organization p. 76-82, 1996

23. Da Villa G, Piccinino F, Scolastico C, Fusco M., Piccinino R, SEPE. Longterm epidemiological survey of hepatitis $B$ virus infection in a hyperendemic area (Afragola, southern Italy) : results of a pilot vaccination project. Research in Virololgy 149: 263-270, 1998.

24. Degos F. Hepatitis C and alcohol. Journal of Hepatology v.3:113-118, 1999.

25. Di Bisceglie AM, Order SE, Klein JL, Waggoner JG, Sjogren MH, Kuo G,
Houghton M, Choo KL, Hoofnagle JH. The role of chronic viral hepatitis in hepatocelular hepatocarcinoma in the United States. American Journal of Gastroenteroly 86: 335-338, 1991.

26. European Association for the Study of the Liver. International consensus conference on hepatitis C, Paris Consensus Statement. Journal of Hepatology 30: 956-961, 1999.

27. European Association for the Study of the Liver. International Consensus on Hepatitis C. Paris, Journal of hepatology $30: 956-961,1999$. Editora Savier, São Paulo p. 26-37, 2003.

28. Ferraz MLG, Yoradjian A, Barbieri A, Lopes EPA, Figueiredo VM, Khouri S, Silva AEB. Critérios diagnósticos de hepatite auto-imune aplicados a pacientes do Ambulatório de Hepatites da Escola Paulista de MedicinaUnifesp. Revista Brasileira de Clínica e Terapêutica 23: 29-32, 1997.

29. Fonseca JC. Epidemiologia da infecção pelo vírus da hepatite $\mathrm{C}$ no Brasilrelatório do grupo de estudo da Sociedade Brasileira de Hepatologia. Gastroenterologia Endoscopia Digestiva 18 ( supl) : s3-s8, 1999.

30. Gomes CS, Lopes R0. Perfil da alfa-fetoproteína nas hepatopatias crônicas. Trabalho de Conclusão de Curso de Medicina, Universidade Federal do Pará, Belém- PA, 2001.

31. Gonçalves CS, Pereira FEL, Gayotto LCC. Hepatocellular carcinoma in Brasil: report of a national survey (Florianópolis, SC, 1995). Revista do Instituto de Medicina Tropical de São Paulo 39: 165-170, 1997.

32. Gregorio GV, Portmann B, Reid F, Donaldson PT, Doherty DG, McCartney M, Mowat AP, Vergani D, Mieli-Vergani. G. Autoimmune Hepatitis in Childhood: A 20-Year Experience. Hepatology 25:541-547,1997.

33. Kew MC, Houghton M, Choo Ql, Kuo G. Hepatitis C virus antibodies in southern African blacks with hepatocellular carcinoma. Lancet 335: 873$874,1990$.

34. Khokhar, N. Spectrum of chronic liver disease in a tertiary care hospital. Journal Park Medical Association 52: 56-58, 2002.

35. Johnson, PJ and Mcfarlane, IA. Meeting report: International Autoimmune Hepatitis Group. Hepatology.18: 998-1005, 1993.

36. Liaw YF, Tai DI., Chu CM, Lin DY, Sheen IS, Chen IJ, Pao, CC. Early detection of hepatocellular carcinoma in patients with chronic B hepatitis. A prospective study. Gastroenterology 90: 263-267, 1986.

37. Margolis HS, Alter JH, Hadler SC. Hepatitis B: evolving epidemiology and implications for control. Seminary of Liver Diseases 11: 84-92, 1991.

38. Mincis, M. Patogenia e fisiopatologia da doença hepática alcoólica. Fatores de risco para o dano hepático pelo etanol. In resumos do XIII Congresso Brasileiro de Hepatologia, Florianópolis p. 177,1995.

39. Miranda ECBM. Estudo clínico-epidemiológico em carcinoma hepatocelular na Amazônia Oriental. Gastroenterologia Endoscopia Digestiva 16:165,1997.

40. Miranda, ECBM. Carcinoma hepatocelular e as infecções pelos vírus das hepatites B e C na Amazônia Oriental - Estudo clínico, sorológico e de biologia molecular. Dissertação apresentada ao Curso de Mestrado em Medicina Tropical da Universidade Federal do Pará, Belém-PA, 1999.

41. Miranda ECBM, Bensabath G, Soares MCP, Amaral ISA, Moia LJMP, Conde SRSS, Barbosa MS, Araújo MTF, Pinheiro MCN, Cruz EM. Pesquisa de ácido nucléico viral HBV-DNA e HCV-RNA por PCR em pacientes com diagnóstico de carcinoma hepatocelular na Amazônia Oriental. In resumos do XV Congresso da Associação Latino - Americana para 0 Estudo do Fígado ALEF, São Paulo p. s95, 1998.

42. Miranda ECBM, Moia LJMP, Amaral ISA, Barbosa MSB, Conde SRSS, Santos ALR, Mendes LMS, Soares MCP, Araújo MTF. Estudo comparativo dos aspectos epidemiológicos das infeccões pelos vírus das hepatites $\mathrm{B}$ e $\mathrm{C}$ na Amazônia Oriental. In resumo do XVI Congresso Brasileiro de Hepatologia, Vitória p. s69, 2001.

43. Moia LJMP, Conde SRSS, Barbosa MSB, Amaral ISA, Miranda ECBM, Soares MCP, Araújo MT, Demachki S. Aspectos etiológicos da doença hepática crônica no sexo feminino, em um hospital de referência. In resumos do XVI Congresso Brasileiro de Hepatologia, Vitória p. s77, 2001.

44. Moia LJMP, Soares MCP, Bensabath G, Amaral ISA, Miranda ECBM, Conde SRSS, Barbosa MSB, Araújo MT, Demachki S. Estudo clínico-epidemiológico de 11 casos de doenças hepática crônica anti-HD positivos na Amazônia 
Oriental Brasileira. In resumos do XV Congresso Brasileiro de Hepatologia, Rio de Janeiro p. s71, 1999.

45. Moia LJMP, Soares MCP, Bensabath G, Amaral ISA, Miranda ECMM, Conde SRSS, Barbosa MSB, Araújo MT, Demachki S. Estudo clínico-epidemiológico de 25 casos de doença hepática crônica anti-HD positivos na Amazônia Oriental Brasileira. In: XV Congresso Brasileiro de Hepatologia, 2003, Recife. Gastroenterologia Endoscopia Digestiva XVI Congresso Brasileiro de Hepatologia 18 ( supl) p. S71, 2003.

46. Moreira CC, Teixeira AC, Soares MCP, Barros VLRS, Alves MM, Silva CAM, Cartágenes P. Hepatotoxicidade (Cróton cajucara Benth) em hamsterresultados preliminares. In resumos do XV Congresso Brasileiro de Hepatologia, Rio de Janeiro, p. s30, 1999.

47. Park YM, Kim IS, Lee CD, Kim, BS. Seroprevalence of antibody against hepatitis $\mathrm{C}$ virus (anti-HCV) in various groups of individuals in Korea. Gastroenterology Japanese 26: S159-S163, 1991.

48. Pinheiro MCN, Araújo R, Araújo MTF, Cruz EM, Blanco AC, Bensabath G, Soares ACP, Amaral ISA, Barbosa MS, Miranda ECBM. Freqüência do anticorpo do vírus $\mathrm{Cem}$ portadores de hepatopatias crônicas. In resumos do XXX Congresso da Sociedade Brasileira de Medicina Tropical, Salvador p. 99, 1994.

49. Porta G. Hepatite Auto-imune. In: Mincis, M., Gastroenterologia \& Hepatologia: Diagnóstico e Tratamento. São Paulo: Lemos-Editorial. p. 637 647.1997
50. Santos ALR, Mendes LMS. Perfil epidemiológico das infecções pelos vírus das hepatites B e C no Hospital da Santa Casa de Misericórdia do Pará Trabalho de Conclusão de curso de Medicina Universidade do Estado do Pará, Belém-Pa, 2000.

51. Shiratori $Y$, Shiina S, Inamura M, Kato N, Kanai F, Okudaira T, Teratani T, Tohgo G, Toda N, Ohashi M, Ogura K, Niwa Y, Kawabe T, Omata M. Characteristic difference of hepatocellular carcinoma between hepatitis B and C viral infection in Japan. Hepatology 22: 1027-1033, 1995.

52. Soares MCP, Amaral ISA. Polycistic echinococcosis by E. vogeli in the Amazon region. Journal of Hepatology 28: 908, 1998.

53. Soares MCP, Teixeira LS, Cartágenes PRB, Moia LJMP, Conde SRSS, Bensabath G. Subtipos do vírus da hepatite C ( $\mathrm{HCV}$ ) em diferentes grupos expostos na Amazônia Oriental Brasileira. In resumos do XV Congresso da Associação Latino- Americana para 0 Estudo do Fígado - ALEF, São Paulo, p. $s 65,1998$.

54. Souto FJD. Distribuição da hepatite B no Brasil: atualização do mapa epidemiológico e proposições para seu controle. Gastroenterologia Endoscopia Digestiva 18: 143-150, 1999.

55. Takano S, Yokosuka 0, Imazeki F Tagawa M., Omata M. Incidence of hepatocellular carcinoma in chronic hepatitis B and C: A prospective study of 251 patients. Hepatology 21: 650-655, 1995. 